\title{
Quantum storage and manipulation of heralded single photons in atomic memories based on electromagnetically induced transparency
}

\author{
Pin-Ju Tsai $\odot,{ }^{1,2}$ Ya-Fen Hsiao, ${ }^{2,3}$ and Ying-Cheng Chen $\odot^{2,4, *}$ \\ ${ }^{1}$ Department of Physics, National Taiwan University, Taipei 10617, Taiwan \\ ${ }^{2}$ Institute of Atomic and Molecular Sciences, Academia Sinica, Taipei 10617, Taiwan \\ ${ }^{3}$ Molecular Science Technology Program, Taiwan International Graduate Program, Academia Sinica and National Central University, \\ Taipei 10617, Taiwan \\ ${ }^{4}$ Center for Quantum Technology, Hsinchu 30013, Taiwan
}

(Received 10 July 2019; accepted 4 June 2020; published 28 July 2020)

\begin{abstract}
We demonstrate the storage and manipulation of heralded single photons generated from a cavity-enhanced spontaneous parametric down-conversion (SPDC) source in the atomic quantum memory based on electromagnetically induced transparency. We show that nonclassical correlations are preserved between the heralding and the retrieved photons after storage process. By varying the intensity of the coupling field during retrieval process, we further demonstrate that the waveform or bandwidth of the single photons can be manipulated and the reduction of the nonclassical correlation between the photon pairs can be compensated. Our SPDC photon pairs are generated in a single pair of longitudinal cavity modes, which not only reduces the experimental complexity arising from external filtering but also increases the useful photon generation rate. Our results can be scaled up with ease and thus lay the foundation for future realization of large-scale applications in quantum information processing.
\end{abstract}

DOI: 10.1103/PhysRevResearch.2.033155

\section{INTRODUCTION}

Quantum memories are devices that can store and retrieve photonic quantum states on demand [1]. The ability of quantum memories to synchronize probabilistic events makes them a key component in quantum repeaters for long-distance quantum communication [2], linear-optics-based quantum computation [3], and enhancing the multiphoton generation rate [4]. Intensive efforts and progress have been made in the development of high-performance quantum memory [5]. While many of these works used the weak coherent laser pulses, some recent experiments have demonstrated the quantum storage of true single photons [6-13]. For the realization of quantum memories, single photons with suitable properties for storage are crucial. Single photons can be produced on demand by single atoms inside a cavity [14-16] or in a heralded way through spontaneous Raman transitions [17] or four-wave mixing [18-21] in atomic ensembles. One advantage of such single photons is that their frequencies are right on the atomic transition and their bandwidths are comparable to the linewidth of atomic transition, allowing efficient interaction between photons and atoms. However, the disadvantage is the relatively complicated setup, which is cumbersome to scale up for large-scale experiments.

\footnotetext{
*chenyc@pub.iams.sinica.edu.tw

Published by the American Physical Society under the terms of the Creative Commons Attribution 4.0 International license. Further distribution of this work must maintain attribution to the author(s) and the published article's title, journal citation, and DOI.
}

Photon pairs generated from SPDC process with nonlinear crystals are widely used in quantum information processing. Heralded single photons (HSPs) can be produced from such photon pairs in which the detection of one (idler) photon heralds the production of its twin (signal photon). Applications of SPDC-based HSP storage to quantum repeaters [22] or to enhance the multiphoton rate [4] have been proposed. Cavityenhanced SPDC has been developed to generate bright photon pairs with a bandwidth comparable to the bandwidth of atomic memories [23-29]. The storage of such HSPs in quantum memories based on atomic ensembles [6,8] and rare-earthdoped crystals has been demonstrated $[7,10]$. However, the photon pairs used in all of these works were multilongitudinal cavity modes. External etalon filters stabilized to the atomic transition frequency are needed to filter out the unwanted modes. This not only complicates the experimental setup, but also reduces the useful generation rate. In a recent work [29], we developed a bright, single-longitudinal-mode photonpair source by cavity-enhanced SPDC. Based on this, we demonstrate the quantum storage and manipulation of HSPs in atomic quantum memories based on electromagnetically induced transparency (EIT) with cold atoms. Nonclassical correlation between the heralding and retrieved photons is demonstrated. By varying the coupling field intensity during retrieval, the single-photon waveform or bandwidth and the nonclassical feature can be manipulated.

This work demonstrates the manipulation of the waveform of true single photons by EIT memory. This bandwidth manipulation is essential for linking quantum nodes of different bandwidths in a quantum network [30]. If retrieved with a coupling beam of different wavelength, it could be used as a quantum frequency converter to bridge quantum nodes made by 
TABLE I. Comparison of quantum storage of heralded single photons generated by cavity-enhanced SPDC source. SE: storage efficiency, ST: storage time; EIT: electromagnetically induced transparency; AFC: atomic frequency comb.

\begin{tabular}{lccccc}
\hline \hline Group & Platform & Protocol & SE $(\%)$ & ST $(\mu \mathrm{s})$ & $g_{s, i}^{(2)}$ \\
\hline Akiba [6] & Cold Rb atom & EIT & 14 & 0.25 & 7.7 \\
Clausen [7] & Nd: $\mathrm{Y}_{2} \mathrm{SiO}_{5}$ & AFC & 21 & 0.025 & 30 \\
Zhang [8] & Cold $\mathrm{Rb}$ atom & EIT & 9.8 & 0.2 & 10 \\
Rieländer [10] & Pr: $\mathrm{Y}_{2} \mathrm{SiO}_{5}$ & AFC & 11 & 1.5 & 11 \\
Seri [35] & Pr: $\mathrm{Y}_{2} \mathrm{SiO}_{5}$ & AFC & 12 & 1.5 & 61 \\
Seri [36] & Pr: $\mathrm{Y}_{2} \mathrm{SiO}_{5}$ & AFC & 8.5 & 3.5 & 72 \\
Our work & Cold Cs atom & EIT & 36 & 0.1 & 7.5 \\
\hline \hline
\end{tabular}

different physical systems in a quantum network [31]. By using a time-dependent coupling pulse during the retrieval process, it could be used to generate single photons with widely tunable waveform [32], as well as a quantum buffer to match the temporal mode of different single-photon sources [33].

We have obtained a storage efficiency of up to $36 \%$, which is currently limited by the bandwidth of photons, the optical depth of the medium, and the coupling-field-induced decoherence due to the off-resonant excitation [34]. Future improvement of the storage efficiency is possible [34]. Although this is not the highest efficiency obtained with single photons [13], it is the highest to date obtained with HSPs based on SPDC (see Table I) [6-8,10,35,36]. Our demonstration of quantum storage and manipulation of HSPs from a compact cavity-SPDC source can be scaled up with ease and lays the foundation for future realization of large-scale protocols in quantum information processing.

\section{EXPERIMENTAL SETUP}

The experimental setup is shown in Fig. 1(a). It is composed of a photon source and a cold-atom system. In the photon source, nondegenerate narrow-band photon pairs are generated by the cavity-enhanced SPDC. We briefly mention essential points here and more details can be referred to [29]. The pump beam, derived from an external cavity diode laser with a wavelength of $407 \mathrm{~nm}$, pumps the type-II periodically poled KTiOPO4 (PPKTP) crystal. The pump power alternates between a high-power $(\sim 40 \mathrm{~mW})$ and a low-power phase $(<0.5 \mathrm{~mW})$ at a repetition rate of $6.7 \mathrm{kHz}$, controlled by an acousto-optic modulator (AOM). During the high- and lowpower phases, the output of the source is in lasing (or called the optical parametric oscillator, OPO) mode and photon-pair mode, respectively. Due to the unbalanced reflectivity of the two cavity end mirrors, about $60 \%$ (40\%) of the OPO power is reflected from (transmitted through) the cavity. After splitting the signal $(852 \mathrm{~nm})$ and idler $(780 \mathrm{~nm})$ components in the transmitted OPO light with a polarizing beam splitter (PBS), the idler is detected by a photodetector which acts as a power monitor for the OPO light. After the PBS, the transmitted signal is further split into two parts by a 50/50 beam splitter. Part of the signal light is detected by a photodetector and its output is used to obtain a demodulated error signal to lock the cavity to resonance through the piezo attached to the cavity output coupling mirror. Another part of the signal
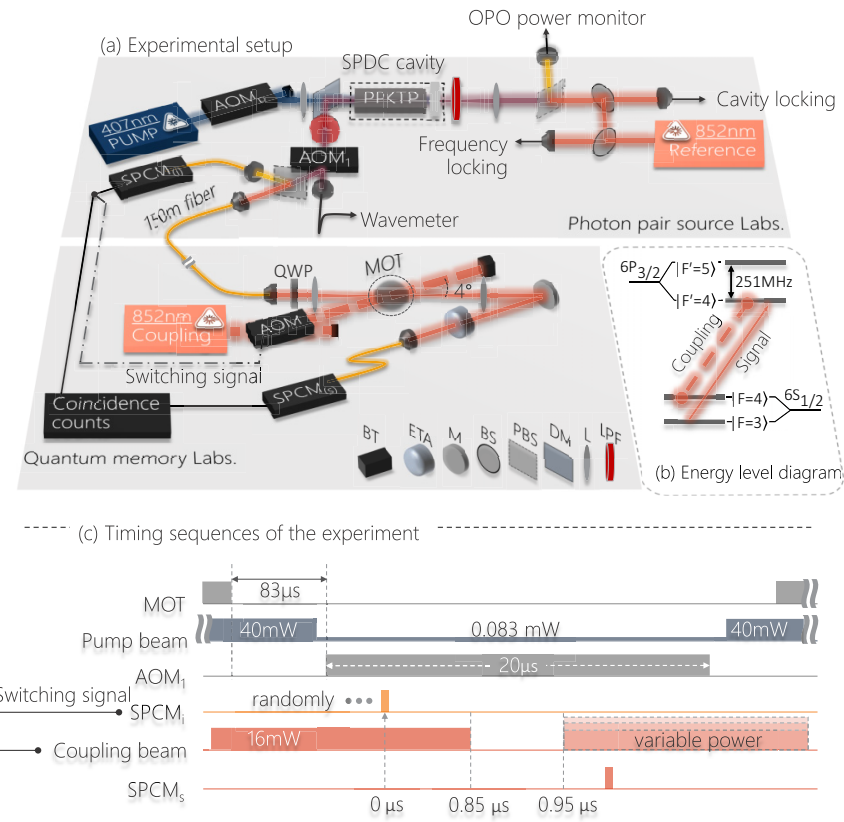

FIG. 1. (a) The experimental setup comprises two parts: the cavity-enhanced SPDC photon-pair source and the cold-atomic quantum memory based on EIT. L, lens; QWP, quarter wave plate; BS, beam splitter; PBS, polarizing beam splitter; LPF, long pass filter; DM, dichroic mirror; ETA: etalon filter; BT: beam trap. (b) Relevant energy levels of ${ }^{133} \mathrm{Cs}$ and laser excitations. (c) Timing sequences of the experiment. The storage process is completed by switching the coupling beam off and on upon triggering from the idler photons.

light interferes with a reference laser. With this beat note, the frequency of the signal is locked to the reference laser with a frequency offset using the simple offset locking scheme [37]. One beam from a master laser, locked to the cesium $D_{2}$ atomic transition, passing through a double-passed AOM is used to injection lock the reference laser. By setting the frequency of that AOM, the frequency of the signal can be tuned around the resonant frequency of the $\left|6 S_{1 / 2}, F=3\right\rangle \rightarrow\left|6 P_{3 / 2}, F=4\right\rangle$ transition.

During the photon-pair output phase, the photon pairs emitted in the backward direction of the cavity are picked up by a dichroic mirror, diffracted by an AOM, and split apart by a PBS. The idler photons are detected by a single-photon counter $\left(\mathrm{SPCM}_{i}\right)$ and act as the trigger to herald the generation of signal photons. The signal photons pass through a 150 -m-long fiber to reach the quantum memory laboratory. The bandwidth of the photon pairs is $6.2 \mathrm{MHz}$, comparable to the natural linewidth of $D_{2}$ line $(5.23 \mathrm{MHz})$. The clustering effect of type-II phase matching and the double-pass pumping results in single-longitudinal-mode operation [25,29,38]. Therefore, there is no need of an external filter to filter out the unwanted frequency modes right after the photon source.

Our atomic quantum memory is based on a magnetooptical trap (MOT) of cesium [39]. We typically trap $\sim 4 \times$ $10^{8}$ cold atoms with a temperature of $180 \mu \mathrm{K}$. To increase the optical depth of the atomic media, we employ the temporally dark MOT and the Zeeman-state optical pumping [34]. The EIT coupling field drives the $\left|6 S_{1 / 2}, F=4\right\rangle \rightarrow$ 
$\left|6 P_{3 / 2}, F=4\right\rangle$ and the signal field drives the $\mid 6 S_{1 / 2}$, $F=3\rangle \rightarrow\left|6 P_{3 / 2}, F=4\right\rangle$ transition, as shown in Fig. 1(b). To characterize the system parameters, the pump power of the photon source is raised up such that the signal output is in lasing mode and becomes a classical pulse of square waveform. By scanning the frequency of the signal field through atomic resonance, we can measure the EIT transmission spectrum and determine the optical depth of the medium by spectral fitting [34]. The typical optical depth of the atomic clouds is around $55 \pm 10$. By switching off the coupling field, the signal photons can be written into the collective atomic spin wave [40]. This EIT memory provides an avenue for manipulation of the stored signal photons by configuring the coupling parameter during the retrieval process $[31,41,42]$.

It is crucial to filter out the coupling beam and other unwanted stray light during the quantum storage experiment. Multiple techniques are used to minimize the background noise including the introduction of a $4^{\circ}$ angle between the coupling and signal beams and blocking the coupling beam by a beam trap. After passing through the MOT cell, the signal beam propagates along a long beam path $(\sim 6 \mathrm{~m})$ with four irises to further block the coupling light. The signal beam then passes through a bandpass filter (Sermock LL01-852-12.5) and an etalon filter (Quantaser FPE001). After coupling into a single-mode fiber, the signal beam is then detected by the single-photon counter. The overall extinction ratio for the coupling beam is $129.1 \mathrm{~dB}$. The overall collection efficiency of the signal light is $21 \%$, which is defined as the power ratio between that on the single-photon counter and the output after passing through a long fiber of $150 \mathrm{~m}$. Details of the transmission efficiency for the components along the beam path include $76 \%$ for a polarizer, $85.4 \%$ for some lenses and the MOT cell, $50.0 \%$ for the etalon, $90 \%$ for the bandpass filter, and $72 \%$ for the coupling into a single-mode fiber.

The timing sequences for the quantum storage experiment are shown in Fig. 1(c). The measurement is run at a repetition rate of $8 \mathrm{~Hz}$. In each cycle, the pump beam of the SPDC source is switched to the low-power phase for photon pair generation, after turning off the MOT cooling beams for $83 \mu \mathrm{s}$. $\mathrm{AOM}_{1}$ is then turned on for $20 \mu$ s to release the photon pairs. Upon triggering by the idler photons, the coupling beam is turned off to store the signal photons for $100 \mathrm{~ns}$ and is turned back on with a variable intensity to retrieve the signal photons. We use a digital oscilloscope (Rohde \& Schwarz RTO-1014) to conduct the coincidence measurement and data statistics. The 150 -m-long fiber for the signal photons induces a $\sim 800 \mathrm{~ns}$ propagation delay which allows for a grace time window to compensate the finite response time of the electronics to turn off the coupling beam.

\section{RESULTS}

\section{A. Characterization of the photon source}

Before employing the storage, we characterize the properties of the photon pairs by measuring the Glauber correlation function $G_{s, i}^{(2)}(\tau)$, which is defined by $\left\langle\hat{a}_{i}^{\dagger}(t+\tau) \hat{a}_{s}^{\dagger}(t) \hat{a}_{s}^{\dagger}(t) \hat{a}_{i}(t+\tau)\right\rangle$, where $\tau$ is the time delay between the arrival of the signal and idler photons at the detector. Figure 2(a) depicts the measured $G_{s, i}^{(2)}(\tau)$ right after
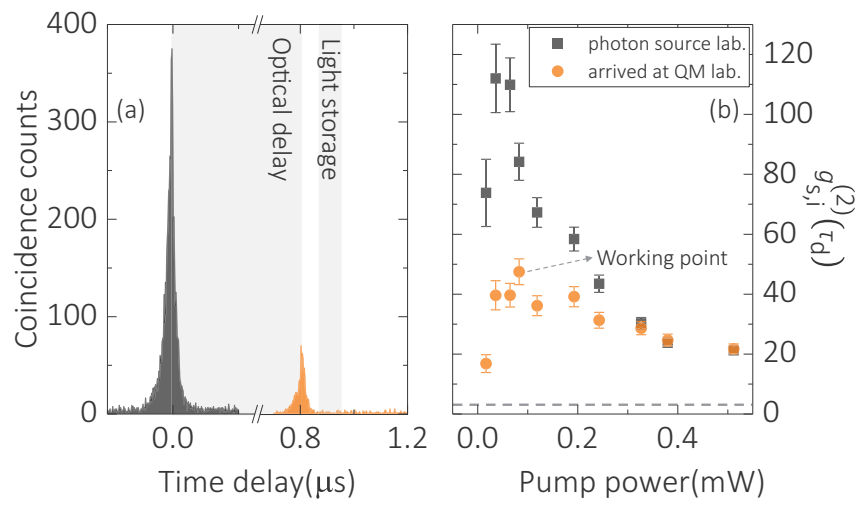

FIG. 2. (a) Biphoton correlation function right after the photon source (red) and in the quantum memory laboratory (orange). The pump power is $0.083 \mathrm{~mW}$. The $\sim 800$-ns delay time in these two cases is due to the propagation delay in a $150-\mathrm{m}$ fiber, an etalon filter, and a $\sim 6$-m free-space path. (b) The normalized cross-correlation function versus the pump power right after the photon source (black squares) and in the quantum memory laboratory (red circles). The dashed line represents the classical limit of 2 .

the photon source and in the quantum memory laboratory after passing through a 150-m-long fiber. The total detection time window is $500 \mathrm{~ns}$ with each time bin of $5 \mathrm{~ns}$. The corresponding detection rates right at those two places are 1.10 and $0.24 \mathrm{kHz}$, respectively. The fiber and other optical components induce an attenuation of the signal photons. The overall collection efficiency of the signal photons in the quantum memory laboratory is $\sim 21 \%$. By analyzing the full width at half-maximum (FWHM) of $G_{s, i}^{(2)}(\tau)$, the correlation time $T_{c}(25 \mathrm{~ns})$ and the bandwidth of the photon pairs can be determined (6.2 MHz) [38].

Furthermore, the normalized cross-correlation function $g_{s, i}^{(2)}=p_{s, i} /\left(p_{s} p_{i}\right)$ which quantifies the nonclassical feature of the photon pairs can be determined by normalizing $G_{s, i}^{(2)}(\tau)$. The parameter $p_{s, i}$ is the probability of the coincidental detection of signal and idler photons and $p_{s}\left(p_{i}\right)$ is the probability of detecting the signal (idler) photons. We determine $g_{s, i}^{(2)}\left(\tau_{d}\right)$ at the peak of the biphoton waveform by

$$
g_{s, i}^{(2)}\left(\tau_{d}\right)=\frac{N_{s, i}\left(\tau_{d}\right)}{\left\langle N_{s}\right\rangle}
$$

where $N_{s, i}\left(\tau_{d}\right)$ denotes the number of coincidences in the 5 -ns time bin at the peak $\left(\tau_{d}\right)$ of the biphoton waveform and $\left\langle N_{s}\right\rangle$ denotes the average of the uncorrelated coincidence counts within 100-ns time window far away from the biphoton waveform. The coincidence histogram is collected for 30000 idler trigger events. The total time for data collection is around $1.5 \mathrm{~h}$.

In order to operate the photon source at optimum condition, the $g_{s, i}^{(2)}$ is measured for various pump powers, as shown in Fig. 2(b). The maximum $g_{s, i}^{(2)}$ appears at a pump power of 0.036 and $0.083 \mathrm{~mW}$ with a value of $\approx 110$ and $\approx 47$ right after the photon source and in the quantum memory laboratory, respectively. These values are all above the nonclassical bound of 2 [43]. In Fig. 2, the reduction of $g_{s, i}^{(2)}$ detected at the memory laboratory is mainly limited by the leakage of stray light into the detector, although we have performed 
serious light shielding. Except this noise source, the leakage photons during the optical parametric oscillator (OPO) phase into the SPDC phase is another limiting factor for $g_{s, i}^{(2)}$. More discussions on the noise are described in Appendix A.

\section{B. Quantum storage and manipulation}

We then facilitate the storage of HSPs by loading cold atoms. In order to make sure that almost all of the biphoton waveform can be compressed into the EIT memory, we first check whether the group delay time of the classical signal pulse is long enough [34]. The pump power of the photon source is raised up such that the signal output is in lasing mode and becomes a classical pulse of square waveform. This allows us to conveniently adjust the locking frequency of the signal photons to EIT resonance. We choose a coupling power such that the ratio of group delay time of the signal pulse to $T_{c}$ $(\sim 25 \mathrm{~ns})$ is $\sim 3$ [34]. Once this is done, the photon source is set back to the photon-pair mode for quantum storage experiment.

To model the data, we quantitatively discuss the evolution of the biphoton correlation function $G_{s, i}^{(2)}(\tau)$ after passing through an EIT memory based on the Heisenberg-Langevin equation (see Appendix $\mathrm{C}$ for more information). The physical meaning of the result is clear if the problem is analyzed in the frequency domain. The conclusion is that $G_{s, i}^{(2)}(\tau)$ is just its initial frequency component times the EIT medium response function and transformed back to the time domain. Equivalently, $G_{s, i}^{(2)}(\tau)$ can be calculated by solving the MaxwellBloch equations with its initial temporal waveform as the input. There are two additional terms contributing to the background of $G_{s, i}^{(2)}(\tau)$ in the theory. One is due to the accidental measurement of the photon pairs. The other is due to the spontaneous decay, which is negligible at the strong-coupling limit. In addition, technical factors such as the leakage of coupling beam and stray light into the single-photon counter and the detector dark count may contribute to the background of $G_{s, i}^{(2)}(\tau)$.

Figure 3(a) depicts the result of slow light with an energy transmission of $52 \%$ at an optical depth of $\sim 50$ and a group delay of $75 \mathrm{~ns}$. The relatively low transmission efficiency is mainly due to the finite ground-state decoherence rate $(\gamma)$ caused by multiple reasons. The off-resonant excitation of the coupling field to a nearby cycling transition, the imperfect compensation of the stray magnetic field, and the residual Doppler broadening all contribute to a relatively large $\gamma$ of $0.09 \Gamma$ [34], which is determined by a numerical fitting to the data. More discussions on the memory loss are described in Appendix B. There is a noticeable stretching of the biphoton correlation function in the time domain, due to the finite EIT bandwidth. Effectively, the EIT medium narrows the spectral bandwidth of the HSPs and acts as a frequency filter. The filtering effect is more significant for single photons generated by the cavity-SPDC setup due to its Lorentzian spectral profile, which has relatively slow-decayed spectral tails. The estimated bandwidth of the slow light is $4.0 \mathrm{MHz}$ by evaluating the FWHM width of the Fourier-transformed spectrum of the simulated $G_{s, i}^{(2)}(\tau)$. This bandwidth is narrower than that of the input photons as expected.

The efficiency for the retrieved case of Fig. 3(c) is 36\%, lower than the $52 \%$ of the slow light case. The additional

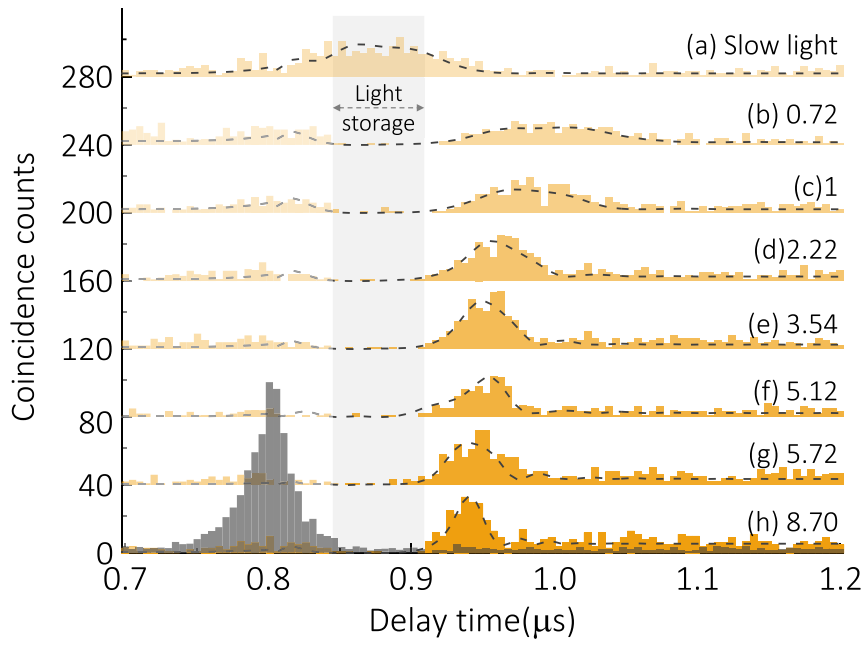

FIG. 3. Biphoton correlation function for various coupling powers in the retrieval process. In case (a), the coupling beam is continuous on with a power of $P_{w}=16 \mathrm{~mW}$. This is the slow light case without storage. In all other cases, the signal photons are stored for $100 \mathrm{~ns}$ and retrieved with a different coupling power. The power ratio between the reading to writing coupling field $\xi_{r / w}$ is also shown in the figure, which ranged from 0.72 to 8.70 for the cases (b) to (h). The black dashed lines indicate the results of the theoretical calculation.

efficiency reduction is due to the leakage of the signal waveform during storage, caused by a relatively slow switching time $(\sim 60 \mathrm{~ns})$ of the coupling field and the choice of a coupling intensity for a tradeoff between minimizing the leakage of the pulse tails and maximizing the slow light transmission [34]. More details are discussed in Appendix B. The nonslowed component in trace (c) of Fig. 3 is the leakage part. Our simulation considering both factors supports this interpretation, as shown in the dashed line of Fig. 3(c). Although there is still a large room to improve the efficiency, the achieved efficiency of $36 \%$ is the highest to date for the storage of heralded single photons generated by the SPDC source. Table I shows a comparison on some recent works demonstrating the storage of HSPs generated by the SPDC source.

EIT memories have been used to manipulate the temporal width and other properties of classical signal pulses by varying the coupling parameters during the retrieval $[31,41,42]$. These ideas can be extended to the quantum regime to manipulate the biphoton correlation function $G_{s, i}^{(2)}(\tau)$. We vary the reading powers $P_{r}$ of the coupling beam to manipulate the retrieved biphoton waveform. We denote the reading-to-writing coupling power ratio as $\xi_{\mathrm{R} / \mathrm{W}}=P_{\mathrm{R}} / P_{\mathrm{W}}$. By choosing $\xi_{\mathrm{R} / \mathrm{W}}>1$, the retrieved $G_{s, i}^{(2)}(\tau)$ can be compressed and the peak value of $G_{s, i}^{(2)}(\tau)$ is larger than that of the slow light case. Figures $3(\mathrm{~b})-3(\mathrm{~h})$ show various traces with different $\xi_{\mathrm{R} / \mathrm{w}}$.

In Fig. 4(b), we demonstrate the bandwidth control of the HSPs based on the data of Fig. 3. The corresponding storage efficiencies are shown in Fig. 4(a), indicating an exponential decay relation versus $\xi_{\mathrm{R} / \mathrm{W}}$, but not a constant as expected in an ideal EIT system [44]. This is due to the off-resonant excitation of the coupling field to the nearby excited state 


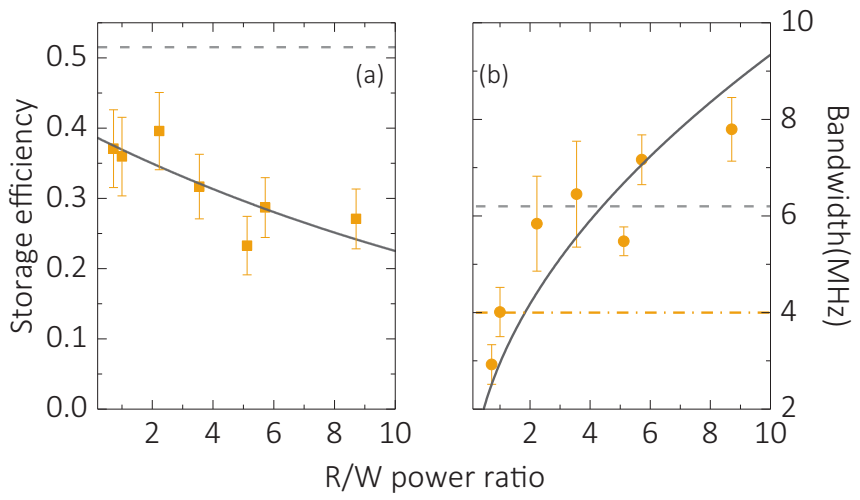

FIG. 4. (a) The storage efficiency versus the read-to-write (R/W) coupling power ratio $\xi_{\mathrm{R} / \mathrm{W}}$. The solid line is an exponential fitting of the data. The dashed line represents the efficiency of the slow light case. (b) The bandwidth of the biphotons versus $\xi_{\mathrm{R} / \mathrm{W}}$. As $\xi_{\mathrm{R} / \mathrm{W}}$ increases, the bandwidth of the biphotons increases. The solid line is a fitting curve with a relation of $2.95 \sqrt{\xi_{\mathrm{R} / \mathrm{w}}}$. The yellow dashed dotted (gray dashed) line represents the bandwidth of the biphotons for the slowed (input) photons.

(e.g., $6 P_{3 / 2}, F=5$ state), which induces a multiphoton decay channel and thus a coupling-intensity-dependent ground-state decoherence rate [34]. If the EIT memory is operated in cesium $D_{1}$ line, this problem will be significantly reduced due to the relatively large detuning for the nearby excited state [34]. The storage efficiency versus $\xi_{\mathrm{R} / \mathrm{W}}$ of Fig. 4(a) can be modeled as $e^{-\gamma_{s} \xi_{\mathrm{R} / \mathrm{w}}}$ [34], where $\gamma_{s}$ is 0.055 as determined by an exponential fitting of the data. Theoretically, $\gamma_{s}$ is related to the optical depth, Rabi frequency of the coupling, and the coupling detuning with respect to the $6 P_{3 / 2}, F=5$ state [34]. By plugging in these parameters, the calculated value is consistent with the fitting parameter to within $10 \%$. The observed bandwidth of the retrieved photons is proportional to $\sqrt{\xi_{\mathrm{R} / \mathrm{W}}}$, instead of $\xi_{\mathrm{R} / \mathrm{W}}$ [45]. This trend is understandable and is explained below. There is a gradual shift in the EIT transparent bandwidth from a linear to a square-root relation as the coupling intensity increases (see Appendix C). The bandwidth of our photon pairs is relatively wide such that the coupling is operated in the high-intensity regime, which explains the $\sqrt{\xi_{\mathrm{R} / \mathrm{W}}}$ trend in the photon bandwidth.

We next address the cross-correlation measurement of the photon pairs $g_{s, i}^{(2)}$. For the slowed and retrieved cases with $\xi_{\mathrm{R} / \mathrm{W}}$ between 0.72 and 8.7 , the peaks $g_{s, i}^{(2)}$ are all larger than the nonclassical threshold value of 2 [43], as shown in Fig. 5. Therefore, the nonclassical feature of the photon pairs is preserved in EIT memories.

Nevertheless, these values of $g_{s, i}^{(2)}$ are significantly lower than those of the input photons. The effects that cause the efficiency reduction also cause a reduction of the $g_{s, i}^{(2)}$. Another important technical factor leading to the reduction of $g_{s, i}^{(2)}$ is the leakage of the coupling beam into the detector (see more discussion in Appendix A). Increasing the storage efficiency of the memory, minimizing the technical losses of all optical components, and increasing the extinction ratio of the filtering setup will increase the nonclassical feature.

As $P_{r}$ increases, the peak $g_{s, i}^{(2)}(\tau)$ first increases, until reaching a maximum and then decreases. The maximum $g_{s, i}^{(2)}(\tau)$

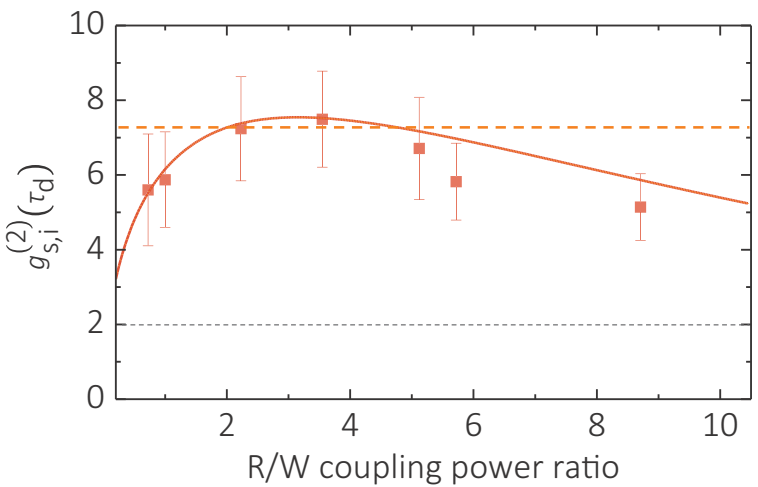

FIG. 5. Manipulation of nonclassical correlation of the retrieved photons. The red squares represent the $g_{s, i}^{(2)}\left(\tau_{d}\right)$ of the retrieval photons with different $\mathrm{R} / \mathrm{W}$ power ratios. The red solid line shows the theoretical fit based on Eq. (2) with the parameters $\gamma_{s} \sim 0.055$, $\alpha=0.43$, and $N_{b}=2.8$. The red dashed line is the cross correlation of the slow light case of 7.2. The gray dashed line depicts the classical limit of $g_{s, i}^{(2)}=2$.

of $\sim 7.5$ appears at $\xi_{\mathrm{R} / \mathrm{W}} \sim 3.5$, which is greater than 5.8 for the case of $\xi_{\mathrm{R} / \mathrm{W}}=1$. This demonstrates that the nonclassical feature can be manipulated by varying the coupling power during retrieval. The peak value of $g_{s, i}^{(2)}$ depends on multiple factors. As discussed previously, it should scale as $\sqrt{\xi_{\mathrm{R} / \mathrm{W}}}$ at the high-coupling intensity regime. Due to the couplingintensity-dependent decoherence rate, there is an additional factor $e^{-\gamma_{s} \xi_{\mathrm{R} / \mathrm{w}}}$. The accidental coincidences with the noise contribute to the background of the coincidence count (denoted as $N_{b}$ ) and thus the degradation of $g_{s, i}^{(2)}\left(\tau_{d}\right)$. The leakage from the coupling beam also contributes to the background coincidence, which is proportional to the coupling power and is denoted as a constant $\alpha$ times $\xi_{\mathrm{R} / \mathrm{W}}$. Based on these discussions, the relation for the peak $g_{s, i}^{(2)}(\tau)$ (which occurs at $\tau=\tau_{d}$ ) versus $\xi_{\mathrm{R} / \mathrm{W}}$ is written as

$$
g_{s, i}^{(2)}\left(\tau_{d}\right)=\frac{N_{s, i} \sqrt{\xi_{\mathrm{R} / \mathrm{W}}} e^{-\gamma_{s} \xi_{\mathrm{R} / \mathrm{w}}}}{\alpha \xi_{\mathrm{R} / \mathrm{W}}+N_{b}},
$$

where $N_{s, i}\left(N_{b}\right)$ denotes the coincidence (background) count within a given integration time. As shown in Fig. 5, the fit curve based on this relation agrees well with the data.

\section{CONCLUSION}

In summary, we demonstrate the quantum storage and manipulation of HSPs generated from the cavity-SPDC source. The bandwidth and nonclassical correlation of the photon pairs can be manipulated by varying the coupling intensity during the retrieval process. It should be noted that the continuous-wave pumped SPDC cavity generates biphotons correlated in frequency, which could degrade their performance in the applications of entanglement swapping and polarization-based qubit gate $[8,46]$. However, this issue can be remedied by using the pulsed pump, as that demonstrated in Ref. [8]. With that in mind, this development lays the foundation for the realization of large-scale protocols in quantum information processing, such as quantum repeaters and enhancing the multiphoton generation rate. 

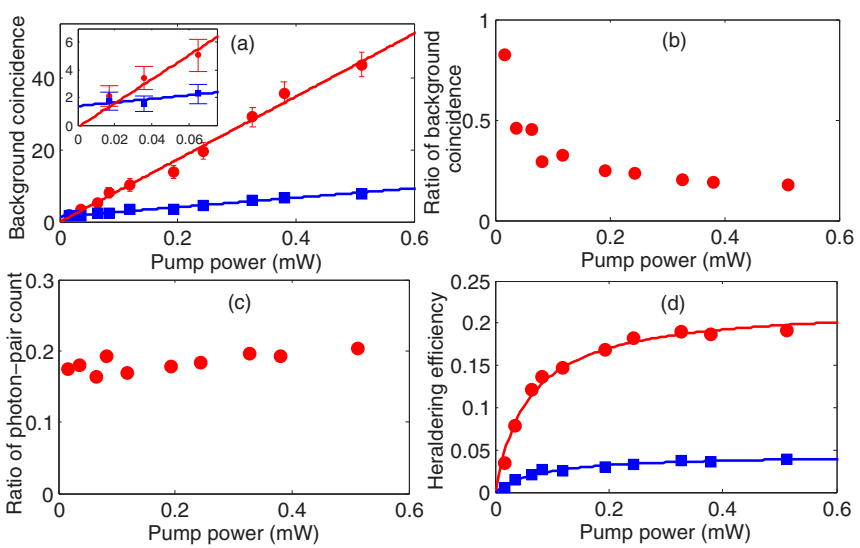

FIG. 6. More data analysis on Fig. 2. (a) Average background coincidence count versus pump power within a time bin of $5 \mathrm{~ns}$. The data of red circle (blue square) are taken at the place of photon source (quantum memory laboratory), and the same symbols apply for data in (d). The total idler trigger event is 30000 . Inset shows the data for low pump power. (b) The ratio of the background coincidence count for that measured at the quantum memory laboratory to that right after the photon source. (c) The ratio of photon-pair count measured at those two places. (d) The heralding efficiency versus pump power measured at those two places. The solid lines are the fitting curves of Eq. (A1).

\section{ACKNOWLEDGMENTS}

This work was done as part of a collaborative project under the Science Vanguard Program and Academic Summit Program of Ministry of Science and Technology (MOST) of Taiwan. We thank I. A. Yu. and Y. F. Chen for their fruitful discussions. We also thank Y.-Y. Li and S.-L. Huang for technical assistance with fiber fusion. This work was supported by MOST of Taiwan under Grants No. 106-2119-M-001-002, No. 107-2112-M-001-003, and No. 107-2745-M-007-001. We also thank the support from NCTS ECP1 and the Center for Quantum Technology from the Featured Areas Research Center Program of Ministry of Education of Taiwan.

P.-J. T. and Y.-F. H. contributed equally to this work.

\section{APPENDIX A: CHARACTERIZATION OF THE NOISE}

In this Appendix, we discuss the noise sources in the coincidence measurement in details. From Fig. 2, it shows that a factor of $\sim 5$ loss leads to a significant reduction of $g_{s, i}^{(2)}$, especially at low pump power. To learn why such a behavior, we do more analysis on the data of Fig. 2. Figure 6(a) depicts the average background coincidence count within one time bin versus the pump power for that detected right after the photon source and that in the quantum memory laboratory. The trend is a linear relation and this is expected since the multiphoton accidental coincidence is proportional to the pump power. The solid lines are the linear fitting curves. The $y$-axis intersect of the fitting curve for the data detected at the memory laboratory is 1.38 , suggesting the existence of a noise source even at zero pump power. The dark count of the detector is $\sim 100 \mathrm{~Hz}$, which contributes to only $\sim 0.015$ in the $y$-axis intersect and cannot explain the observation. In Fig. 6(b), we plot the ratio of the background coincidences for that detected at the memory laboratory to that right after the photon source. At high pump power, the ratio is nearly 0.2 , which is the overall transmission efficiency between those two places. The ratio starts to rise up at low pump power, which also suggests the existence of additional noises detected at the quantum memory laboratory. As a reference, we also plot the ratio of the total photon-pair count detected at those two places versus the pump power, as shown in Fig. 6(c). The photon-pair ratio is almost a constant $(\sim 0.183 \pm 0.013)$ for all pump power. Combining these analyses, it is evident that the additional noise detected at the quantum memory laboratory accounts for the reduction of $g_{s, i}^{(2)}$. We suspect that the leakage of the stray light from all used lasers into the single-photon detector is the major noise source, although we have done a careful light shielding on the detector.

In Fig. 6(d), we also plot the pump power dependence of the heralding efficiency $\eta_{H}$, determined by summing up the total photon-pair count divided by the total idler triggers $\left(N_{i}=\right.$ 30000 ). If we categorize the idler trigger events into two parts, one is due to the unpaired noise $N_{n}$ and the other is due to the paired photons $N_{i, s}$ with an efficiency $\eta_{i}$ for the idler detection. Since the number of photon pairs is proportional to the pump power, we can denote $\eta_{i} N_{i, s}=k P$ with $P$ the pump power in $\mathrm{mW}$ and $k$ the number of detected, paired idler photons per $\mathrm{mW}$. By denoting the detection efficiency for signal photon as $\eta_{s}$, the heralding efficiency can be written as

$$
\eta_{H}=\frac{\eta_{s} \eta_{i} N_{s, i}}{\eta_{i} N_{s, i}+N_{n}}=\frac{\eta_{s} k P}{k P+N_{n}} .
$$

This equation fits the data very well, as shown in Fig. 6(d). It can be seen that when increasing the pump power such that $k P \gg N_{n}, \eta_{H}$ approaches to $\eta_{s}$ and when decreasing the pump power such that $k P<N_{n}, \eta_{H}$ approaches to zero quickly. The fitting parameters $\left\{\eta_{s}, k, N_{n}\right\}$ for the data detected right after the photon source and in the memory laboratory are $\{0.2208,4328,257.0\}$ and $\{0.0445,3741,296.6\}$, respectively. The ratio of $\eta_{s}$ for the latter case to the former case is 0.202 , which is nearly the same as the transmission efficiency determined by the strong classical signal light.

To learn more about the noise source, we turn off the lasers for the MOT and EIT experiment and conduct the coincidence measurement again at the quantum memory laboratory. We measure the coincidence for two cases with the signal photons passing or bypassing an etalon filter. The results of $g_{s, i}^{(2)}$ and average background coincidence count in a 5-ns time bin versus the pump power are shown in Figs. 7(a) and 7(b), respectively. The ratio of the transmission efficiency for the case of passing to bypassing the etalon is $\sim 0.63$. From Fig. 7(b), the zero pump power intersects of the $y$ axis for the two cases are 0.64 and 0.71 , respectively. These values are around a factor of 2 lower than that of Fig. 2. This implies that the stray light leakage does contribute a significant part $(\sim 50 \%)$ of the noise. Due to the relative close transmission efficiency and stray light leakage for the two measurement cases, compared to that of Fig. 2, the difference in $g_{s, i}^{(2)}$ of Fig. 7 is not as significant as that of Fig. 2. The maximum $g_{s, i}^{(2)}$ for that passing the etalon is smaller than that bypassing the etalon, unlike that of Ref. [10], suggesting that there is likely negligible broadband noise in our photon source. Otherwise, 


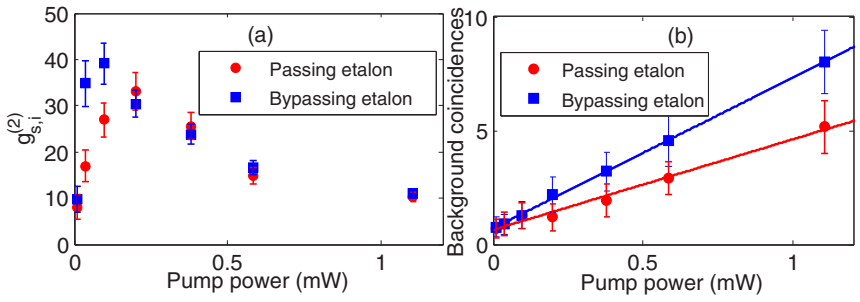

FIG. 7. (a) $g_{s, i}^{(2)}$ versus the pump power for signal photons passing (red circle) and bypassing (blue square) the etalon. The data were taken in the quantum memory laboratory and with all trapping, repumping, and EIT lasers off. (b) The average background coincidence count measured in a time bin of $5 \mathrm{~ns}$ versus the pump power. The total idler trigger event is 30000 .

the situation should be the opposite since the broadband noise photon should be attenuated by the etalon. Even though we have minimized the stray light leakage, there is still a certain amount of zero-pump-power background coincidence that cannot be explained by the detector dark count. This leads us to suspect that the light leakage of the OPO operation phase into the SPDC phase might be one of the noise sources.

To check this possibility, we measure the coincidence count versus the delay of the gate time after the photon source is switching from the OPO to the SPDC phase. With a gate time window of $5 \mu \mathrm{s}$ for collecting the coincidence event and a total idler trigger of 30000 , the results are shown in Fig. 8. Surprisingly, the counts exponentially decay to a steady-state value of the SPDC phase with a long $e^{-1}$ time constant of $6.67 \mu \mathrm{s}$, compared to the $\sim 25$-ns coherence time of the photon pairs. The gain narrowing effect of the lasing condition for the OPO operation phase may explain for such a long time constant. Figure 9(a) depicts the $g_{s, i}^{(2)}$ versus the pump power for three gate time of 0-10, 10-20, and 20-30 $\mu$ s. Obviously, the maximum $g_{s, i}^{(2)}$ increases significantly for the data taken at a later gate time window. Figure 9(b) depicts the average background coincidence count within a 5-ns time bin versus the pump power. The $y$-axis intersect at zero pump power for the data taken at a later time window reduces significantly compared to that taken at the earliest one. The OPO leakage

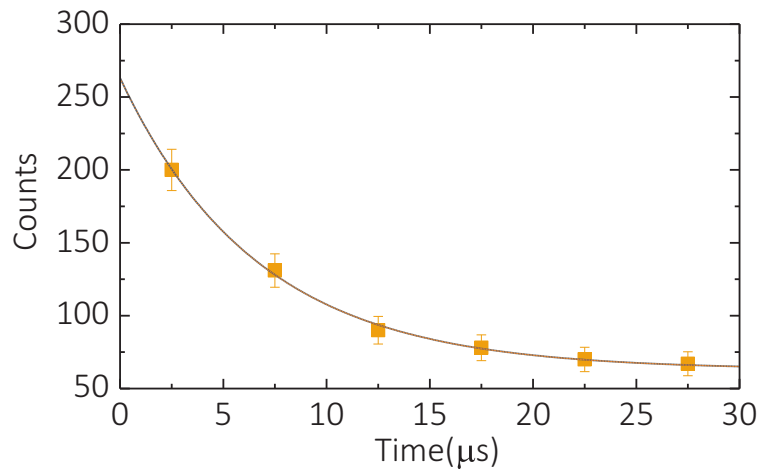

FIG. 8. The coincidence count versus the time after switching from the OPO to the SPDC operation phase. Time zero is actually $3.68 \mu \mathrm{s}$ after the switching time. The $e^{-1}$ time constant is $6.67 \mu \mathrm{s}$. Each data point is collected within a gate time of $5 \mu$ s for a total idler trigger event of 30000 .
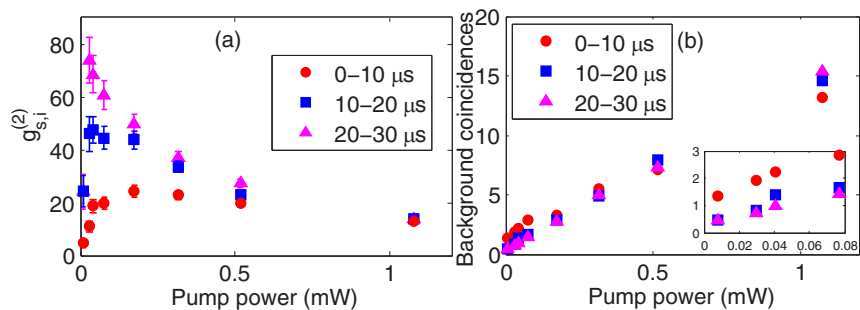

FIG. 9. (a) $g_{s, i}^{(2)}$ versus the pump power for data collected in a $10-\mu$ s time window with a different delay time after switching from the OPO to SPDC operation phase. (b) The average background coincidence count in a time bin of $5 \mathrm{~ns}$ versus the pump power for the three gate times. Inset is the magnified portion for low pump power. Since the data are congested, the error bars with $1 \sigma$ standard deviation are not shown for clarity. The total idler trigger event is 30000 .

into the SPDC phase is certainly one serious noise that one should be cautious when using our cavity-SPDC scheme. It is necessary to choose a time window starting at a long enough delay after switching from the OPO to the SPDC phase to conduct the single-photon experiment. Our data of Figs. 2, 3, and 7 were taken at a delay time of $8.68 \mu \mathrm{s}$. This delay is not quite enough and it is expected that this noise may limit the maximum value of $g_{s, i}^{(2)}$ for those situations. The data set of Fig. 9 were measured at the place right after the photon source.

When one loads the cold atoms to conduct the quantum storage experiment, there is one additional noise source due to the leakage of the coupling light into the detector. Based on the data of Fig. 3, Fig. 10 depicts the background coincidence count versus the read-to-write coupling power ratio, which is basically a linear relation as expected. The leakage of the coupling beam is another important limiting factor to reduce $g_{s, i}^{(2)}$. That is why one has to use the sophisticated setups to filter out the coupling light. The zero-couplingpower intersect is 2.793, which is larger than that without loading the cold atoms. This may be due to the higher stray light leakage into the detector when all the lasers are in operation.

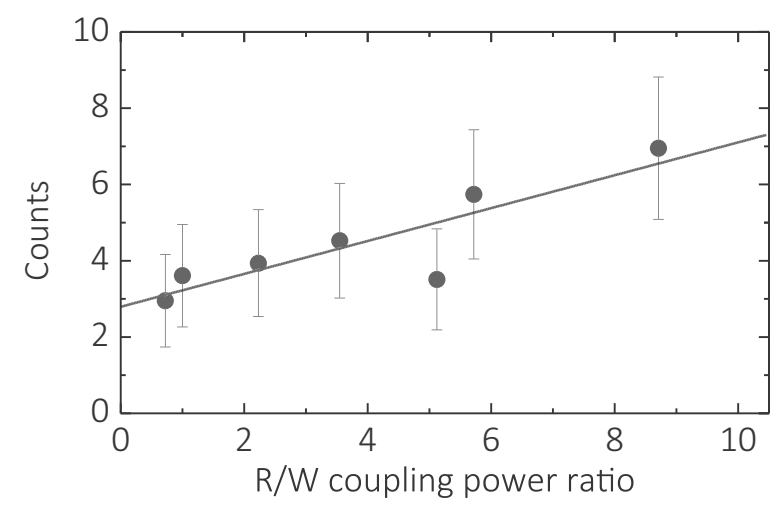

FIG. 10. Background coincidence count within a time bin of $5 \mathrm{~ns}$ versus the R/W coupling power ratio. Data are collected for 30000 idler trigger events. The solid line is a linear fit to the data with a $y$-axis intersect of 2.79 . 


\section{APPENDIX B: DISCUSSION ON THE MEMORY LOSS}

In this Appendix, we discuss in more details the memory loss. The finite ground-state decoherence rate (denoted as $\gamma$ ) significantly affects the storage efficiency. There are many factors contributed to the finite decoherence rate. In this work, we have to use a relatively strong-coupling intensity $\left[\Omega_{c}=\right.$ 5.3 $\Gamma$ for the case of Fig. 3(c)] to broaden the EIT transparent bandwidth since the bandwidth of our single photons is relatively wide $(\sim 6.2 \mathrm{MHz})$. As demonstrated in [34], the EIT system implemented in the $D_{2}$ line of cesium suffers from the coupling-intensity-dependent ground-state decoherence rate due to the off-resonant excitation of the coupling field to the nearby transition. This mechanism causes an energy loss for the signal pulse whenever the coupling field is on, either during the storage or retrieval process.

Based on the numerical simulation for the slow light data of Fig. 3(a), we estimate an overall ground-state decoherence rate of $0.09 \Gamma$, which is quite large compared to that in [34]. The simulation curve matches the data well with an efficiency of $52 \%$. If the decoherence rate were zero, the calculated slow light efficiency would be $80 \%$, which highlights the significant role of the finite ground-state decoherence rate. We estimate that a decoherence rate of $0.059 \Gamma$ is due to the off-resonant excitation of the coupling field using the formula in [34]. In the manipulation cases in which the intensity of the reading coupling field is stronger than that of the writing coupling field, the signal experiences even more loss, as shown in Fig. 4(a). Again, this reflects the loss due to the coupling-intensity-dependent ground-state decoherence rate during retrieval process.

Another major factor of the decoherence rate comes from the stray magnetic field. The stray magnetic field of the MOT system used for this work was not well compensated as we did in another MOT system used for the work of Ref. [34]. Also, we use a relatively large angle $\left(\sim 4^{\circ}\right)$ between the signal and coupling beam in order to reduce the coupling leakage into the single-photon counter. The larger angle induces a shorter effective wavelength for the collective spin wave which is easier to dephase due to atomic thermal motion [47]. A combination of these two factors causes an exponential decay for the efficiency versus storage time with a relatively short $e^{-1}$ time constant of $1.2 \mu \mathrm{s}$. Based on this, we estimate a decoherence rate of $0.027 \Gamma$ due to these two factors.

Furthermore, the temporal waveform of single photons is an exponential decay curve such that its spectral distribution is a Lorentzian profile. This is different from the Gaussian classical pulse used in [34], which also has a Gaussian distribution in the frequency domain. The Lorentzian spectral profile has a relatively long tail compared to the Gaussian one which may experience more absorption with a given EIT transparent bandwidth. If one takes a closer look at the temporal distribution of the coincidence counts of the slow light case, it is clear to see that its temporal waveform becomes more like a Gaussian distribution. This means that its spectral profile has been modified by the EIT medium with its long spectral tails being absorbed more than its central region as mentioned above. The temporal width of the slowed single-photon waveform becomes much wider than that of the input one, which also reflects the narrowed spectral width of the slowed single photons.

For the case of storage and retrieval (compared to the slow light case), there are two more factors that cause additional losses. First, the temporal stretching (or spectral narrowing) effect for the signal photons with an exponential decay waveform is more significant when passing through an EIT medium. If one chooses a weaker-coupling field, the group velocity is smaller such that one can almost compress the whole signal pulse into the medium. However, the EIT transparent bandwidth also becomes narrower such that the slow light transmission is relatively low. On the contrary, when one chooses a stronger-coupling field, the slow light transmission is larger but the group velocity may be not small enough such that the front and rear tails may leak the medium during the storage process. There is an optimum coupling intensity such that the overall efficiency for the stored light is optimal. With our experimental conditions, a certain percentage of leakage in the tails is required in order to obtain an optimal retrieval efficiency. The second factor is a technical reason that increases the leakage percentage of the signal waveform. The coherence time of the biphoton is $\sim 25 \mathrm{~ns}$, which is shorter than the switching time of the electronics to turn off the coupling field $(\sim 60 \mathrm{~ns})$. This slow switching-off time causes more front tail leaking out of the medium. In the trace (c) of Fig. 3, it is clear that part of the front edge of the single-photon waveform leaks because it is not slowed. Our numerical simulation supports this observation by taking both factors into consideration, as shown in the dashed line of Fig. 3(c). From the data, we estimate that $\sim(15 \pm 3) \%$ of the pulse energy is lost due to the leakage. This explains why the efficiency of the retrieved signal $(\sim 36 \%)$ is less than that of the slow light case $(\sim 52 \%)$.

\section{APPENDIX C: THEORETICAL BACKGROUND}

\section{Theoretical background of EIT quantum memory}

In this Appendix, we consider the full quantum theory of a quantized signal field propagating through an EIT quantum memory (QM). As shown in Fig. 1(b), the EIT QM is constituted of a three-level atomic system that contains two ground states of $|F=3(4)\rangle=|g(s)\rangle$ and one excited state $\left|F^{\prime}=4\right\rangle=|e\rangle$. The atomic population is prepared at $|g\rangle$ initially. A strong, classical light couples the $|s\rangle \leftrightarrow|e\rangle$ transition with a Rabi frequency of $\Omega_{c}$. A quantized signal field $\hat{a}_{s}(z, t)$ is on resonance with the $|g\rangle \leftrightarrow|e\rangle$ transition. The equation of motion for the atomic system is given by the Heisenberg-Langevin equations

$$
\begin{array}{r}
\frac{\partial}{\partial t} \hat{\sigma}_{g e}(z, t)=\frac{i}{2} \Omega_{c} \hat{\sigma}_{g s}(z, t)+\frac{i}{2} g_{s} \hat{a}_{s}(z, t) \\
+\left(i \delta_{g e}-\frac{\Gamma}{2}\right) \hat{\sigma}_{g e}(z, t)+\hat{F}_{g e}, \\
\frac{\partial}{\partial t} \hat{\sigma}_{g s}(z, t)=\frac{i}{2} \Omega_{c}^{*} \hat{\sigma}_{g s}(z, t)+\left(i \delta_{g s}-\frac{\gamma_{g s}}{2}\right) \hat{\sigma}_{g s}+\hat{F}_{g s},
\end{array}
$$

where $\delta_{g e}$ and $\delta_{g s}$ denote the one- and two-photon detuning of the signal field, $\hat{\sigma}_{i j}$ is the slowly varying atomic coherence operator, $\hat{F}_{i j}$ is the Langevin noise operator, $g_{s}$ is the coupling constant for atom-field interaction, $\Gamma$ is the spontaneous decay 
rate from $|e\rangle$ to ground states, and $\gamma_{g s}$ is the decoherence rate between $|g\rangle$ and $|s\rangle$. The equation of motion for the signal field propagating in the atomic medium is described by the Maxwell-Schrodinger equation of

$$
\left(\frac{1}{c} \frac{\partial}{\partial t}+\frac{\partial}{\partial z}\right) \hat{a}_{s}(z, t)=\frac{i g_{s} N}{c} \hat{\sigma}_{g e},
$$

where $N$ is the atom number and $c$ is the speed of light in a vacuum. To solve the problem, we first Fourier transform Eqs. (C1)-(C3) to the frequency domain. By solving the coupled equations of Eqs. (C1) and (C2), the atomic coherence can be obtained as follows:

$$
\hat{\sigma}_{g e}(\omega)=\frac{i g_{s}}{2} \frac{d_{g s}}{\mathbf{D}(\omega)} \hat{a}_{s}+\hat{f}(\omega),
$$

where $\mathbf{D}(\omega)=d_{g e} d_{g s}+\left|\Omega_{c} / 2\right|^{2}, \quad d_{i j}=\gamma_{i j} / 2-i\left(\omega+\delta_{i j}\right)$. And $\hat{f}(\omega)=\left(i \Omega_{c} \hat{F}_{g s}+d_{g s} \hat{F}_{g e}\right) / \mathbf{D}(\omega)$. By substituting Eq. (C4) into (C3), the field equation becomes

$$
\frac{\partial}{\partial z} \hat{a}_{s}-\frac{i \omega}{c} \hat{a}_{s}=-\frac{g_{s}^{2} N}{2 c} \frac{d_{g s}}{\mathbf{D}(\omega)} \hat{a}_{s}+\frac{i g_{s} N}{c} \hat{f}(\omega),
$$

and

$$
\begin{aligned}
\hat{a}_{s}(z, \omega) & =\hat{a}_{s}(0, \omega) e^{-\Lambda(\omega) z}+\delta \hat{a}_{s}(z, \omega), \\
\delta \hat{a}_{s}(z, \omega) & =\int_{0}^{z} \frac{i g_{s} N}{c} \hat{f}(\omega) e^{\Lambda(\omega)\left(z^{\prime}-z\right)} d z,
\end{aligned}
$$

where $\Lambda(\omega)=-\frac{i \omega}{c}+\frac{g_{s}^{2} N d_{g s}}{2 c \mathbf{D}(\omega)}$. Equation (C6) shows the evolution of the signal field after interacting with an EIT medium in the frequency domain, which contains two terms. The first term is just the input signal field multiplied by the EIT medium frequency response. This term represents the transmissive and dispersive proprieties of the signal field during its propagation in an EIT medium. The second term $\delta \hat{a}_{s}(z, \omega)$ in the field solution describes the field fluctuations from the atomic system. The field fluctuations can be neglected under the strong-coupling condition since the $\delta \hat{a}_{s}(z, \omega)$ is contributed by the atomic population in the excited state [48,49].

Equation (C6) provides the solution depicting the interaction between the field and EIT QM. By selecting an initial quantum field of $\hat{a}_{s}(0, \omega)$, we can simulate the output fields from the EIT QM. In our case, the signal field is the single photon generated by the cavity-enhanced SPDC. In the next section, we write the field solution for the cavity-SPDC to evaluate the interaction between the SPDC field and EIT QM.

\section{Biphoton correlation function in an EIT medium}

For a cavity-enhanced SPDC under single-mode operation, the signal and idler output fields are given by [38]

$$
\begin{gathered}
\hat{a}_{s}^{\text {out }}(\omega)=A_{s}(\omega) \hat{a}_{s}^{\text {in }}(\omega)+B_{s}(\omega) \hat{a}_{i}^{\text {in } \dagger}\left(-\omega_{i}\right), \\
\hat{a}_{i}^{\text {out } \dagger}\left(-\omega_{i}\right)=B_{i}(\omega) \hat{a}_{s}^{\text {in }}(\omega)+A_{i}(\omega) \hat{a}_{i}^{\text {in } \dagger}\left(-\omega_{i}\right),
\end{gathered}
$$

where $\hat{a}(\omega)=\frac{1}{2 \pi} \int \hat{a}(t) \exp (i \omega t) d t$ is the Fourier transform of the field operator from the time to the frequency domain, and $\omega_{i}=\omega_{\text {pump }}-\omega$. The coefficients in Eqs. (C7) and (C8) are

$$
\begin{gathered}
A_{s, i}(\omega)=\frac{\gamma_{s, i}-\Gamma_{s, i} / 2+i\left(\omega_{, i}-\Omega_{q, r}\right)}{\Gamma_{s, i} / 2-i\left(\omega_{, i}-\Omega_{s, r}\right)}, \\
B_{s, i}(\omega)=\frac{\mp i \kappa \sqrt{\gamma_{s} \gamma_{i}}}{\left[\Gamma_{s} / 2-i\left(\omega-\Omega_{q}\right)\right]\left[\Gamma_{i} / 2+i\left(\omega_{i}-\Omega_{r}\right)\right]},
\end{gathered}
$$

where $\Omega_{q, r}$ is the frequency of the cavity mode of the signal and idler field [38]. For the double-resonance condition of the cavity, we have a relation of $\Omega_{q}+\Omega_{r}=\omega_{\text {pump }}$ and thus $\omega_{i}-$ $\Omega_{r}=\Omega_{q}-\omega$.

In the experiment, the signal photons are sent into the EIT medium. The field operator of the input signal field $\hat{a}_{s}(0, \omega)$ in Eq. (C6) can be replaced by the signal field operator out of the photon source, i.e., $\hat{a}_{s}^{\text {out }}(\omega)$. By replacing $\hat{a}_{s}(0, \omega)$ with $\hat{a}_{s}^{\text {out }}(\omega)$ and combining Eqs. (C7) and (C8), we obtain the signal field operator in an EIT medium as follows:

$$
\hat{a}_{s}^{\mathrm{EIT}}(z, \omega)=\alpha_{s}(z, \omega) \hat{a}_{s}^{\mathrm{in}}(\omega)+\beta_{s}(z, \omega) \hat{a}_{i}^{\mathrm{in} \dagger}\left(-\omega_{i}\right),
$$

where $\alpha_{s}(z, \omega)=A_{s}(\omega) e^{-\Lambda(\omega) z} \cdot \beta_{s}(z, \omega)=B_{s}(\omega) e^{-\Lambda(\omega) z}$. In the experiment, we measure the two-photon correlation function which is

$$
G_{s, i}^{(2)}\left(t_{s}, t_{i}\right)=\left\langle\hat{a}_{i}^{\text {out } \dagger}\left(t_{i}\right) \hat{a}_{s}^{\text {out } \dagger}\left(t_{s}\right) \hat{a}_{s}^{\text {out }}\left(t_{s}\right) \hat{a}_{i}^{\text {out }}\left(t_{i}\right)\right\rangle,
$$

where $t_{s, i}$ is the time when the signal (idler) photon hits the detector. Using Eqs. (C7), (C8), and (C11), the two-photon correlation function for signal photons passing through the EIT medium is thus

$$
G_{s, i}^{(2)}(\tau)=\left|\frac{1}{2 \pi} \int_{-\infty}^{\infty} d \omega \alpha_{s}^{\dagger}(z, \omega) B_{i}(\omega) e^{i \omega \tau}\right|^{2}+\langle n\rangle,
$$

where

$$
\begin{aligned}
\langle n\rangle= & \frac{1}{(2 \pi)^{2}} \int_{-\infty}^{\infty} d \omega\left|B_{i}(\omega)\right|^{2}\left[\int_{-\infty}^{\infty} d \omega\left|\beta_{s}(z, \omega)\right|^{2}\right. \\
& \left.+\iint_{-\infty}^{\infty} d \omega d \omega^{\prime}\left\langle\delta \hat{a}_{s}^{\dagger}(z, \omega) \delta \hat{a}_{s}\left(z, \omega^{\prime}\right)\right\rangle e^{i\left(\omega^{\prime}-\omega\right) t_{s}}\right] .
\end{aligned}
$$

The second term in Eq. (C13) is a time-independent background quantity which contains two terms, as shown in Eq. (C14). The first term is due to the accidental coincidence measurements with nearby photons, which can be suppressed by choosing a lower pump field to avoid too many photon pairs being generated. The second term is due to the spontaneous decay which is negligible under the strong-coupling condition. The main property of the photon-atom interaction is determined by the first term of Eq. (C13). This term is nothing but the Fourier transform of the frequency component of the waveform $A_{s}^{\dagger}(\omega) B_{s}(\omega)$ multiplied by the EIT medium frequency response $e^{-\Lambda(\omega) z}$ and then transforming back to the time domain. To simulate the biphoton waveform after passing through an EIT medium, it can be calculated in the frequency domain by Eq. (C13) with an initial waveform of $A_{s}^{\dagger}(\omega) B_{s}(\omega)$, or it can be calculated using Eqs. (C1)-(C3) with the input pulse of $A_{s}^{\dagger}(\omega) B_{s}(\omega)$ in the time domain. 


\section{Bandwidth of an EIT medium}

Considering the semiclassical model of Eq. (C6), we obtain the transmission of the signal field after passing through an EIT medium by

$$
\begin{aligned}
& T\left(\delta_{g e}\right) \\
& \quad=\exp \{-2 \operatorname{Re}[\Lambda(0)] L\} \\
& \quad=\exp \left[\frac{-\alpha \Gamma\left[\gamma_{g s}\left|\Omega_{c}\right|^{2}+\left(4 \delta_{g s}^{2}+\gamma_{g s}^{2}\right) \Gamma\right]}{\left(\left|\Omega_{c}\right|^{2}+\Gamma \gamma_{g s}-4 \delta_{g e} \delta_{g s}\right)^{2}+\left(2 \delta_{g e} \gamma_{g s}+2 \delta_{g s} \Gamma\right)^{2}}\right],
\end{aligned}
$$

where $\alpha=2 N L g_{s}^{2} / \Gamma c$ is the optical depth. Equation (C15) is the EIT spectrum as a function of one- and two-photon detuning. In a typical experiment, the coupling detuning is set to zero and thus $\delta_{g s}=\delta_{g e}$. Fitting the EIT spectrum to Eq. (C15) allows us to determine the experimental parameters. Based on Eqs. (C15), we can determine the full width at half-maximum (FWHM) of the EIT transparent bandwidth, which is

$$
\Delta \omega_{\mathrm{EIT}}=\sqrt{(x+y)\left[1-\left(1-\frac{y^{2}}{(x+y)^{2}}\right)^{\frac{1}{2}}\right]} \Gamma,
$$

where $x=\alpha /(2 \ln 2)-1 / 2$ and $y=\left|\Omega_{c}\right|^{2} / \Gamma^{2}$. There is a lower limit for the optical depth of $\ln 2$ such that the factor $x$ in Eq. (C16) is greater than zero. This is because the absorption depth needs to surpass 0.5 in order to possibly define the FWHM bandwidth of the transparent window. Based on Eq. (C16), we can roughly divide the $\Delta \omega_{\text {EIT }}$ into two regimes of $x \gg y$ and $x \ll y$, depending on the intensity of the coupling field. For the case of low-coupling intensity $(x \gg y)$, the EIT transparent bandwidth is

$$
\Delta \omega_{\mathrm{EIT}} \approx \frac{y}{\sqrt{2 x}} \Gamma=\sqrt{\frac{\ln 2}{\alpha}} \frac{\left|\Omega_{c}\right|^{2}}{\Gamma},
$$

which is linearly proportional to the coupling intensity and inversely proportional to $\sqrt{\alpha}$. For the case of high-coupling intensity $(y \gg x)$, the EIT transparent bandwidth is

$$
\Delta \omega_{\mathrm{EIT}} \approx\left|\Omega_{c}\right|\left(1-\sqrt{\frac{x}{2 y}}\right) \approx\left|\Omega_{c}\right| .
$$

Equation (C18) shows that the EIT transparent bandwidth is approximately proportional to the $\Omega_{c}$ in the high-coupling intensity regime. This explains the $\sqrt{\xi_{r / w}}$ scaling law for $g_{s, i}^{(2)}\left(\tau_{d}\right)$ in Eq. (2). The dependence of the EIT bandwidth on the coupling intensity is a key point for the control of biphoton waveform and bandwidth.
[1] F. Bussières, N. Sangouard, M. Afzelius, H. de Riedmatten, C. Simon, and W. Tittel, J. Mod. Opt. 60, 1519 (2013).

[2] N. Sangouard, C. Simon, H. de Riedmatten, and N. Gisin, Rev. Mod. Phys. 83, 33 (2011).

[3] P. Kok, W. J. Munro, K. Nemoto, T. C. Ralph, J. P. Dowling, and G. J. Milburn, Rev. Mod. Phys. 79, 135 (2007).

[4] J. Nunn, N. K. Langford, W. S. Kolthammer, T. F. M. Champion, M. R. Sprague, P. S. Michelberger, X.-M. Jin, D. G. England, and I. A. Walmsley, Phys. Rev. Lett. 110, 133601 (2013).

[5] K. Heshami, D. G. England, P. C. Humphreys, P. J. Bustard, V. M. Acosta, J. Nunn, and B. J. Sussman, J. Mod. Opt. 63, 2005 (2016).

[6] K. Akiba, K. Kashiwagi, M. Arikawa, and M. Kozuma, New J. Phys. 11, 013049 (2009).

[7] C. Clausen, I. Usmani, F. Bussières, N. Sangouard, M. Afzlius, and H. de Riedmatten, Nature (London) 469, 508 (2011).

[8] H. Zhang, X.-M. Jin, J. Yang, H.-N. Dai, S.-J. Yang, T.-M. Zhao, J. Rui, Y. He, X. Jiang, F. Yang, G.-S. Pan, Z.-S. Yuan, Y. Deng, Z.-B. Chen, X.-H. Bao, S. Chen, B. Zhao, and J.-W. Pan, Nat. Photonics 5, 628 (2011).

[9] S. Zhou, S. Zhang, C. Liu, J. F. Chen, J. Wen, M. M. T. Loy, G. K. L. Wong, and S. Du, Opt. Express 20, 24124 (2012).

[10] D. Rieländer, K. Kutluer, P. M. Ledingham, M. Gündoğan, J. Fekete, M. Mazzera, and H. de Riedmatten, Phys. Rev. Lett. 112, 040504 (2014).

[11] D. G. England, K. A. G. Fisher, J.-P. W. MacLean, P. J. Bustard, R. Lausten, K. J. Resch, and B. J. Sussman, Phys. Rev. Lett. 114, 053602 (2015).
[12] E. Distante, P. Farrera, A. Padrón-Brito, D. Paredes-Barato, G. Heinze, and H. de Riedmatten, Nat. Commun. 8, 14072 (2017).

[13] Y. Wang, J. Li, S. Zhang, K. Su, Y. Zhou, K. Liao, S. Du, H. Yan, and S.-L. Zhu, Nat. Photonics 13, 346 (2019).

[14] A. Kuhn, M. Hennrich, and G. Rempe, Phys. Rev. Lett. 89, 067901 (2002).

[15] J. McKeever, A. Boca, A. D. Boozer, R. Miller, J. R. Buck, A. Kuzmich, and H. J. Kimble, Science 303, 1992 (2004).

[16] M. Mücke, J. Bochmann, C. Hahn, A. Neuzner, C. Nölleke, A. Reiserer, G. Rempe, and S. Ritter, Phys. Rev. A 87, 063805 (2013).

[17] C. W. Chou, S. V. Polyakov, A. Kuzmich, and H. J. Kimble, Phys. Rev. Lett. 92, 213601 (2004).

[18] S. Du, P. Kolchin, C. Belthangady, G. Y. Yin, and S. E. Harris, Phys. Rev. Lett. 100, 183603 (2008).

[19] R. T. Willis, F. E. Becerra, L. A. Orozco, and S. L. Rolston, Phys. Rev. A 82, 053842 (2010).

[20] L. Zhao, X. Guo, C. Liu, Y. Sun, M. M. T. Loy, and S. Du, Optica 1, 84 (2014).

[21] L. Zhu, X. Guo, C. Shu, H. Jeong, and S. Du, Appl. Phys. Lett. 110, 161101 (2017).

[22] C. Simon, H. de Riedmatten, M. Afzelius, N. Sangouard, H. Zbinden, and N. Gisin, Phys. Rev. Lett. 98, 190503 (2007).

[23] X.-H. Bao, Y. Qian, J. Yang, H. Zhang, Z.-B. Chen, T. Yang, and J.-W. Pan, Phys. Rev. Lett. 101, 190501 (2008).

[24] M. Scholz, L. Koch, R. Ullmann, and O. Benson, Appl. Phys. Lett. 94, 201105 (2009).

[25] C.-S. Chuu, G. Y. Yin, and S. E. Harris, Appl. Phys. Lett. 101, 051108 (2012). 
[26] J. Fekete, D. Rieländer, M. Cristiani, and H. de Riedmatten, Phys. Rev. Lett. 110, 220502 (2013).

[27] M. Rambach, A. Nikolova, T. J. Weinhold, and A. G. White, APL Photonics 1, 096101 (2016).

[28] C.-H. Wu, T.-Y. Wu, Y.-C. Yeh, P.-H. Liu, C.-H. Chang, C.-K. Liu, T. Cheng, and C.-S. Chuu, Phys. Rev. A 96, 023811 (2017).

[29] P.-J. Tsai and Y.-C. Chen, Quantum Sci. Technol. 3, 034005 (2018).

[30] Kent A. G. Fisher, Duncan G. England, J.-P. W. MacLean, P. J. Bustard, K. J. Resch, and B. J. Sussman, Nat. Commun. 7, 11200 (2016).

[31] Y.-F. Chen, P.-C. Kuan, S.-H. Wang, C.-Y. Wang, and I. A. Yu, Opt. Lett. 31, 3511 (2006).

[32] P. Farrera, G. Heinze, B. Albrecht, M. Ho, M. Chávez, C. Teo, N. Sangouard, and H. de Riedmatten,, Nat. Commun. 7, 13556 (2016).

[33] S. Gao, O. Lazo-Arjona, B. Brecht, K. T. Kaczmarek, S. E. Thomas, J. Nunn, P. M. Ledingham, D. J. Saunders, and I. A. Walmsley, Phys. Rev. Lett. 123, 213604 (2019).

[34] Y.-F. Hsiao, P.-J. Tsai, H.-S. Chen, S.-X. Lin, C.-C. Hung, C.-H. Lee, Y.-H. Chen, Y.-F. Chen, I. A. Yu, and Y.-C. Chen, Phys. Rev. Lett. 120, 183602 (2018).

[35] A. Seri, G. Corrielli, D. Lago-Rivera, A. Lenhard, H. de Riedmatten, R. Osellame, and M. Mazzera, Optica 5, 934 (2018).

[36] A. Seri, D. Lago-Rivera, A. Lenhard, G. Corrielli, R. Osellame, M. Mazzera, and H. de Riedmatten, Phys. Rev. Lett. 123, 080502 (2019).
[37] U. Schünemann, H. Engler, R. Grimm, M. Weidemüller, and M. Zielonkowski, Rev. Sci. Instrum. 70, 242 (1999).

[38] C.-S. Chuu and S. E. Harris, Phys. Rev. A 83, 061803(R) (2011).

[39] Y.-F. Hsiao, Y.-J. Lin, and Y.-C. Chen, Phys. Rev. A 98, 033419 (2018).

[40] M. Fleischhauer and M. D. Lukin, Phys. Rev. Lett. 84, 5094 (2000).

[41] A. K. Patnaik, F. L. Kien, and K. Hakuta, Phys. Rev. A 69, 035803 (2004).

[42] Y.-F. Chen, S.-H. Wang, C.-Y. Wang, and I. A. Yu, Phys. Rev. A 72, 053803 (2005).

[43] A. Kuzmich, W. P. Bowen, A. D. Boozer, A. Boca, C. W. Chou, L.-M. Duan, and H. J. Kimble, Nature (London) 423, 731 (2003).

[44] A. V. Gorshkov, A. André, M. Fleischhauer, A. S. Sørensen, and M. D. Lukin, Phys. Rev. Lett. 98, 123601 (2007).

[45] M. D. Lukin, M. Fleischhauer, A. S. Zibrov, H. G. Robinson, V. L. Velichansky, L. Hollberg, and M. O. Scully, Phys. Rev. Lett. 79, 2959 (1997).

[46] T. S. Humble and W. P. Grice, Phys. Rev. A 77, 022312 (2008).

[47] B. Zhao, Y.-A. Chen, X.-H. Bao, T. Strassel, C.-S. Chuu, X.-M. Jin, J. Schmiedmayer, Z.-S. Yuan, S. Chen, and J.-W. Pan, Nat. Phys. 5, 95 (2009).

[48] M. Fleischhauer, U. Rathe, and M. O. Scully, Phys. Rev. A 46, 5856 (1992).

[49] N. Lauk, C. O’Brien, and M. Fleischhauer, Phys. Rev. A 88, 013823 (2013). 